\title{
Corticosteroid therapy for critically ill patients with COVID-19: A structured summary of a study protocol for a prospective meta-analysis of randomized trials
}

Jonathan A. C. Sterne ${ }^{1,2^{*}}$ D, Janet Diaz ${ }^{3}$, Jesús Villar ${ }^{4,5}$, Srinivas Murthy ${ }^{6}$, Arthur S. Slutsky ${ }^{7}$, Anders Perner ${ }^{8}$, Peter Jüni ${ }^{7}$, Derek C. Angus ${ }^{9}$, Djillali Annane ${ }^{10}$, Luciano Cesar Pontes Azevedo ${ }^{11}$, Bin Du ${ }^{12}$, Pierre-Francois Dequin ${ }^{13,14}$, Anthony C. Gordon ${ }^{15}$, Cameron Green ${ }^{16}$, Julian P. T. Higgins ${ }^{1,2,17}$, Peter Horby ${ }^{18}$, Martin J. Landray 19,20,21, Giuseppe Lapadula ${ }^{22}$, Amelie Le Gouge ${ }^{23}$, Marie Leclerc ${ }^{24}$, Jelena Savović1,17, Bruno Tomazini ${ }^{11}$, Balasubramanian Venkatesh ${ }^{25}$, Steve Webb $^{16}$, and John C. Marshall ${ }^{26}$ for the WHO COVID-19 Clinical Management and Characterization Working Group

\begin{abstract}
Objectives: Primary objective: To estimate the effect of corticosteroids compared with usual care or placebo on mortality up to 28 days after randomization. Secondary objectives: To examine whether the effect of corticosteroids compared with usual care or placebo on mortality up to 28 days after randomization varies between subgroups related to treatment characteristics, disease severity at the time of randomization, patient characteristics, or risk of bias. To examine the effect of corticosteroids compared with usual care or placebo on serious adverse events.
\end{abstract}

Study design: Prospective meta-analysis of randomized controlled trials. Both placebo-controlled and open-label trials are eligible.

Participants: Hospitalised, critically ill patients with suspected or confirmed COVID-19.

Intervention and comparator: Intervention groups will have received therapeutic doses of a steroid (dexamethasone, hydrocortisone or methylprednisolone) with IV or oral administration immediately after randomization.

The comparator groups will have received standard of care or usual care or placebo.

Main outcome: All-cause mortality up to 28 days after randomization.

(Continued on next page)

\footnotetext{
* Correspondence: jonathan.sterne@bristol.ac.uk

${ }^{1}$ Population Health Sciences, Bristol Medical School, University of Bristol,

Bristol, UK

${ }^{2} \mathrm{NIHR}$ Bristol Biomedical Research Centre, Bristol, UK

Full list of author information is available at the end of the article
}

(c) The Author(s). 2020 Open Access This article is licensed under a Creative Commons Attribution 4.0 International License, which permits use, sharing, adaptation, distribution and reproduction in any medium or format, as long as you give appropriate credit to the original author(s) and the source, provide a link to the Creative Commons licence, and indicate if changes were made. The images or other third party material in this article are included in the article's Creative Commons licence, unless indicated otherwise in a credit line to the material. If material is not included in the article's Creative Commons licence and your intended use is not permitted by statutory regulation or exceeds the permitted use, you will need to obtain permission directly from the copyright holder. To view a copy of this licence, visit http://creativecommons.org/licenses/by/4.0/. The Creative Commons Public Domain Dedication waiver (http://creativecommons.org/publicdomain/zero/1.0/) applies to the data made available in this article, unless otherwise stated in a credit line to the data. 
(Continued from previous page)

Search methods: Systematic searching of clinicaltrials.gov, EudraCT, the WHO ISRCTN registry, and the Chinese clinical trials registry. Additionally, research and WHO networks will be asked for relevant trials.

Risk of bias assessments: These will be based on the Cochrane RoB 2 tool, and will use structured information provided by the trial investigators on a form designed for this prospective meta-analysis.

Summary of findings: We will use GRADE to assess the certainty of the evidence.

Statistical analyses: Trial investigators will provide data on the numbers of participants who did and did not experience each outcome according to intervention group, overall and in specified subgroups. We will conduct fixed-effect (primary analysis) and random-effects (Paule-Mandel estimate of heterogeneity and Hartung-Knapp adjustment) meta-analyses. We will quantify inconsistency in effects between trials using $1^{2}$ statistics. Evidence for subgroup effects will be quantified by ratios of odds ratios comparing effects in the subgroups, and corresponding interaction p-values. Comparisons between subgroups defined by trial characteristics will be made using randomeffects meta-regression. Comparisons between subgroups defined by patient characteristics will be made by estimating trial-specific ratios of odds ratios comparing intervention effects between subgroups then combining these using random-effects meta-analysis. Steroid interventions will be classified as high or low dose according to whether the dose is greater or less than or equal to 400 mg hydrocortisone per day or equivalent. We will use network meta-analysis methods to make comparisons between the effects of high and low dose steroid interventions (because one trial randomized participants to both low and high dose steroid arms).

PROSPERO registration number: CRD42020197242

Full protocol: The full protocol for this prospective meta-analysis is attached as an additional file, accessible from the Trials website (Additional file 1). To expedite dissemination of this material, the familiar formatting has been eliminated; this Letter serves as a summary of the key elements of the full protocol for the systematic review.

Keywords: COVID-19, Randomised controlled trial, Systematic Review, Corticosteroid, Dexamethasone, Hydrocortisone, Methylprednisolone, Mortality, Meta-analysis

\section{Supplementary information}

Supplementary information accompanies this paper at https://doi.org/10. 1186/s13063-020-04641-3.

\section{Additional file 1.}

\section{Acknowledgements}

We gratefully acknowledge the efforts of all trial investigators, and the patients who consented to participation. We thank Agnes Sagfors and staff of the W2O group for administrative and communications support.

\section{Authors' contributions}

All authors contributed to drafting the protocol for the prospective metaanalysis. The author(s) read and approved the final manuscript.

\section{Funding \\ Funding for administrative and communications support was provided by the World Health Organisation. No other specific funding for the prospective meta-analysis was received. Dr Diaz contributed to the design of the study. World Health Organisation staff contacted trial investigators to request their participation in this prospective meta-analysis and that they share outcome data.}

\section{Availability of data and materials}

All data provided by the trials, including summary outcome data overall and in the specified subgroups, will be included in supplementary material of the report of the prospective meta-analysis.

\section{Ethics approval and consent to participate}

All trials received ethics approval. No ethics approval was required for this secondary data analysis.

\section{Consent for publication}

Not applicable

\section{Competing interests}

Jonathan A C Sterne is partly funded by the NIHR Bristol Biomedical Research Centre. Jesus Villar received a Research grant from MAQUET (Sörna, Sweden) to perform a clinical trial on mechanical ventilation. Srinivas Murthy is a member of the REMAP-CAP International Trial Steering Committee. Arthur Slutsky is Co-PI of the Dexamethasone in COVID ARDS study and is supported by grants from the Canadian Institutes of Health Research (CIHR). Anders Perner is the sponsorinvestigator of the COVID STEROID trial, which is funded by the Novo Nordisk Foundation and supported by Pfizer. Peter Jüni serves as unpaid member of steering groups or executive committees of trials funded by Abbott Vascular, Astra Zeneca, Biotronik, Biosensors, St. Jude Medical, Terumo and The Medicines Company, has received research grants to the institution from Appili Therapeutics, Astra Zeneca, Biotronik, Biosensors International, Eli Lilly, The Medicines Company, and honoraria to the institution for participation in advisory boards and/or consulting from Amgen, Ava and Fresenius, but has not received personal payments by any pharmaceutical company or device manufacturer. Derek $C$ Angus is a member of the REMAP-CAP International Trial Steering Committee and chair of the REMAP-CAP Corticosteroid Domainspecific Working Group. Djillali Annane has been involved as an investigator and in the steering committee for CAPE-COVID that was publicly funded by a grant from the French Ministry of Health; as an investigator, chief investigator for France, and member of the Steering committee for REMAP-CAP, he received a grant from the French Ministry of health to support French sites that participated to REMAP-CAP corticosteroids domain. He has not received any personal payment from any private or public entities. Luciano C P Azevedo is the PI for the Codex trial, which received donation of dexamethasone from Aché Pharmaceuticals. Bin Du is the PI for the corticosteroids therapy in adult patients with COVID-19 and ARDS (Steroids-SARI) trial, which was funded by a grant from the Ministry of Science and Technology; and has 
received research grants from Chinese Academy of Medical Sciences (Chinese Academy of Medical Sciences (CAMS) Innovation Fund for Medical Sciences (CIFMS) [2016-12 M-1-014 and 2019-I2M-1-001]). Pierre Francois-Dequin is the principal investigator of the CAPE COVID trial which was funded by a program of the French Ministry of Health. Anthony C Gordon is the UK Chief Investigator and a member of the REMAP-CAP International Trial Steering Committee, is funded by an NIHR Research Professorship (RP-2015-06-18) and is supported by the NIHR Imperial Biomedical Research Centre. Outside of this work he has received consulting fees from GlaxoSmithKline and Bristol Myers Squibb paid to his institution. Cameron Green is a member of the REMAP-CAP International Trial Steering Committee. Julian P T Higgins is partly funded by the NIHR Bristol Biomedical Research Centre and partly funded by the NIHR Applied Research Collaboration West (NIHR ARC West) at University Hospitals Bristol and Weston NHS Foundation Trust. Peter Horby is co-chief investigator of the RECOVERY trial of potential treatments for COVID-19 (funded by UKRI and NIHR). He is an employee of the University of Oxford with salary supported by the Wellcome Trust, National Institute of Health Research, and the Medical Research Council. Martin Landray is co-chief investigator of the RECOVERY trial of potential treatments for COVID-19 (funded by UKRI and NIHR), research funding to University of Oxford received from Novartis, Boehringer Ingelheim, and Merck Sharp \& Dohme, infrastructure and core funding received from Health Data Research UK, NIHR Oxford Biomedical Research Centre, UK Biobank Ltd, MRC Population Health Research Unit, and British Heart Foundation Centre for Research Excellence. He is an employee of University of Oxford with salary supported by Li Ka Shing Foundation, Health Data Research UK, NIHR Oxford Biomedical Research Centre, Wellcome Trust, and National Health Service. Jelena Savović is partly funded by the NIHR Applied Research Collaboration West (NIHR ARC West) at University Hospitals Bristol and Weston NHS Foundation Trust. Balasubramanian Venkatesh is a member of the trial management committee of the COVID STEROID trial. Steve Webb is supported by St John of God Healthcare and this work was supported by grants from National Health and Medical Research Council (Australia), Minderoo Foundation, Health Research Council of New Zealand. John Marshall reports honoraria from AM Pharma (DSMB Steering Committee Chair), receives funding from $C I H R$, is the Canadian PI for REMAP-CAP, is a member of the REMAP-CAP International Trial Steering Committee, and is co-chair of the WHO Working Group on Clinical Characterization and Management. The other authors report no conflicts of interest.

\section{Author details}

${ }^{1}$ Population Health Sciences, Bristol Medical School, University of Bristol, Bristol, UK. ${ }^{2} \mathrm{NIHR}$ Bristol Biomedical Research Centre, Bristol, UK. ${ }^{3}$ Clinical Unit, Health Emergencies Programme, World Health Organization, Geneva, Switzerland. ${ }^{4}$ Research Unit, Hospital Universitario Dr. Negrin Las Palmas de Gran Canaria, Las Palmas, Spain. ${ }^{5} \mathrm{CIBER}$ de Enfermedades Respiratorias, Instituto de Salud Carlos III, Madrid, Spain. 'Department of Pediatrics, University of British Columbia, Vancouver, Canada. ${ }^{7}$ Applied Health Research Centre, Li Ka Shing Knowledge Institute of St. Michael's Hospital, Department of Medicine, University of Toronto, Toronto, Canada. ${ }^{8}$ Department of Intensive Care, Rigshospitalet, Copenhagen, Denmark. ${ }^{9}$ Department of Critical Care Medicine, University of Pittsburgh School of Medicine, Pittsburgh, PA, USA. ${ }^{10}$ Department of Intensive Care, Raymond Poincaré Hospital (APHP), School of Medicine Simone Veil, University Paris Saclay -UVSQ, Paris, France. ${ }^{11}$ Critical Care and Emergency Medicine, Hospital Sirio Libanês, São Paulo, Brazil. ${ }^{12}$ Peking Union Medical College Hospital, Beijing, China. ${ }^{13}$ Médecine Intensive - Réanimation, INSERM CIC1415, CHRU de Tours, Tours, France. ${ }^{14}$ CRICS-TriGGERSep network, Centre d'Etude des Pathologies Respiratoires, Université de Tours, Tours, France. ${ }^{15}$ Division of Anaesthetics, Pain Medicine \& Intensive Care, Imperial College London, London, UK. ${ }^{16}$ Australian and New Zealand Intensive Care Research Centre, School of Public Health and Preventive Medicine, Monash University, Melbourne, Australia. ${ }^{17} \mathrm{NIHR}$ Applied Research Collaboration (ARC) West, University Hospitals Bristol and Weston NHS Foundation Trust, Bristol, UK. ${ }^{18}$ Nuffield Department of Medicine, University of Oxford, Oxford, UK. ${ }^{19}$ Nuffield Department of Population Health, University of Oxford, Oxford, UK. ${ }^{20}$ MRC Population Health Research Unit, University of Oxford, Oxford, UK. ${ }^{21} \mathrm{NIHR}$ Oxford Biomedical Research Centre, Oxford University Hospitals NHS Foundation Trust, Oxford, UK. ${ }^{22}$ Division of Infectious Diseases, San Gerardo Hospital, ASST Monza,
Monza, Italy. ${ }^{23} \mathrm{CIC}$ INSERM 1415 - CHRU de Tours, Hôpital Bretonneau, Tours, France. ${ }^{24}$ Délégation à la Recherche Clinique et à I'Innovation, CHRU de Tours, Tours, France. ${ }^{25}$ George Institute for Global Health, University of New South Wales, Sydney, Australia. ${ }^{26} \mathrm{Li}$ Ka Shing Knowledge Institute, St. Michael's Hospital, University of Toronto, Toronto, Canada.

Received: 11 July 2020 Accepted: 29 July 2020

Published online: 24 August 2020

\section{Publisher's Note}

Springer Nature remains neutral with regard to jurisdictional claims in published maps and institutional affiliations.
Ready to submit your research? Choose BMC and benefit from:

- fast, convenient online submission

- thorough peer review by experienced researchers in your field

- rapid publication on acceptance

- support for research data, including large and complex data types

- gold Open Access which fosters wider collaboration and increased citations

- maximum visibility for your research: over $100 \mathrm{M}$ website views per year

At BMC, research is always in progress.

Learn more biomedcentral.com/submissions 